\title{
LAMOST observations in the Kepler field
}

\author{
P. De Cat ${ }^{1, a}$, J.N. Fu ${ }^{2}$, X.H. Yang ${ }^{2}$, A.B. Ren ${ }^{2}$, A. Frasca ${ }^{3}$, J. Molenda-Żakowicz ${ }^{4}$, G. Catanzaro ${ }^{3}$,
} R.O. Gray ${ }^{5}$, C.J. Corbally ${ }^{6}$, J.R. Shi ${ }^{7}$, H.T. Zhang ${ }^{7}$, and A.L. Luo ${ }^{7}$

1 Royal Observatory of Belgium, Brussels, Belgium

2 Beijing Normal University, Beijing, China

3 INAF (Osservatorio Astrofisico di Catania), Catania, Italy

4 Instytut Astronomiczny, Uniwersytet Wrocławski, Wrocław, Poland

5 Appalachian State University, Boone, North Carolina (USA)

6 Vatican Observatory Research Group, University of Arizona, Tucson, Arizona (USA)

7 Key Lab for Optical Astronomy, National Astronomical Observatories, Chinese Academy of Sciences, Beijing 100012, China

\begin{abstract}
The Large Sky Area Multi-Object Fiber Spectroscopic Telescope (LAmost) at the Xinglong observatory in China is a new 4-m telescope equipped with 4,000 optical fibers. In 2010, we initiated the LAMOST-Kepler project. We requested to observe the full field-of-view of the nominal Kepler mission with the LAmost to collect low-resolution spectra for as many objects from the KIC 10 catalogue as possible. So far, 12 of the 14 requested LAMOsT fields have been observed resulting in more than 68,000 low-resolution spectra. Our preliminary results show that the stellar parameters derived from the LAMOsT spectra are in good agreement with those found in the literature based on high-resolution spectroscopy. The Lamost data allows to distinguish dwarfs from giants and can provide the projected rotational velocity for very fast rotators.
\end{abstract}

\section{Introduction}

The space mission Kepler has been designed to detect Earth-like planets around solar-type stars by the transit method [1]. It was launched on 2009 March 7 at 03:49:57.465 UTC and has been collecting ultraprecise photometry with a spectral bandpass from $400 \mathrm{~nm}$ to $850 \mathrm{~nm}$ for a fixed field-of-view (FoV) of 105 square degrees in the constellations Lyra and Cygnus. In May 2013, a second of the four reaction wheels of the Kepler spacecraft failed, preving the telescope from precisely pointing toward stars. Even though ultrahigh precision photometry can no longer be collected, the project is continued as the $\mathrm{K} 2$ mission and the legacy of the Kepler photometry is a pure goldmine for asteroseismic studies of all types of pulsating stars.

The success of asteroseismic studies has been shown to depend crucially on the availability of basic stellar parameters, such as the effective temperature $\left(T_{\text {eff }}\right)$, surface gravity $(\log g)$, metallicity $([\mathrm{Fe} / \mathrm{H}])$, and the stellar rotation rate $(v \sin i)$ (e.g. [2]). These parameters can not be derived from the Kepler data as is the case for data with multi-colour photometry or spectroscopy. Before the launch of the Kepler spacecraft, there was a large effort to derive the stellar parameters from Sloan photometry for potential Kepler targets. These are available in the Kepler Input Catalogue (KIC 10; [3]). Unfortunately, not for all stars KIC 10 stellar parameters are available. Moreover, the precision of the $T_{\text {eff }}$ and $\log g$ in KIC 10 is generally too low for asteroseismic modelling, especially for hot and peculiar stars (e.g. [4]). Also, additional information on the stellar chemical composition and rotation rate is lacking. Hence, to exploit the Kepler data best, additional ground-based spectroscopic data are required (e.g. [5]).

\footnotetext{
a e-mail: Peter.DeCat@oma. be
}

This is an Open Access article distributed under the terms of the Creative Commons Attribution License 4.0, which permits unrestricted use, distribution, and reproduction in any medium, provided the original work is properly cited. 

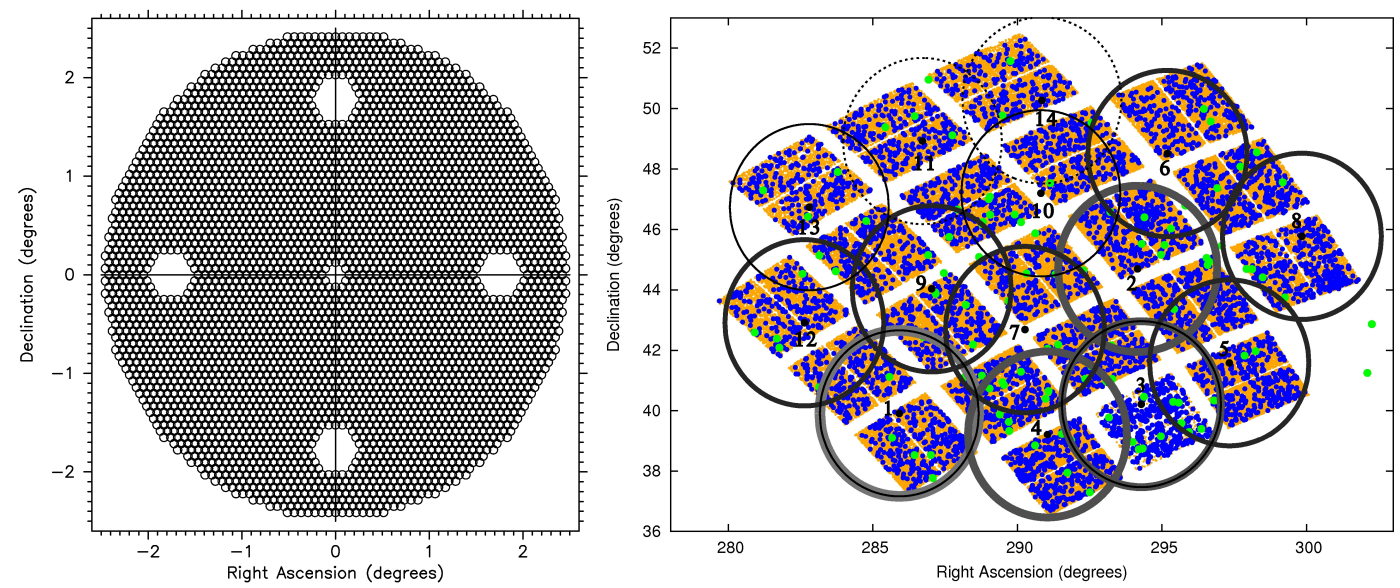

Fig. 1. Left: The 4,000 fibers are homogeneously distributed on the focal plane except for the position of active optics wavefront sensor (central hole) and 4 Guiding CCD cameras (off-centre holes). Right: Representation of the targets of scientific interest in the FoV of the Kepler mission. The black dots refer to the centres of the 14 LK-fields that cover the Kepler FoV (cf. Table 1). We use green for STANDARD targets, blue for Kasc targets and orange for PLANET targets. The scientific importance is reflected in the size of the symbols. The LK-fields that have been observed in 2011, 2012, 2013, and 2014 are indicated by the circles drawn with a full line going from thick to thin and from grey to black, respectively. The two fields that remain unobserved so far are given with a black dashed line. (colour representation only available online)

The Large Sky Area Multi-Object Fiber Spectroscopic Telescope (also called the Guo Shou Jing Telescope) is a unique astronomical instrument located at the Xinglong observatory (China) that combines a large aperture (4-m telescope) with a wide FoV (circular with diameter $5^{\circ}$ ) [6]. The focal surface is covered with 4,000 optical fibers (Fig. 1, left) connected to 16 sets of multi-objective optical spectrometers with 250 optical fibers each [7]. Each spectrometer has two CCD cameras to obtain low resolution spectra $(R \simeq 1800)$ in two wavelength regions (blue arm: $370-590 \mathrm{~nm}$; red arm: $570-900 \mathrm{~nm}$ ). The active optics technique is used to control the reflecting corrector [8]. The LAMOsT has a quasi-meridian transit configuration capable of tracking the motion of celestial objects during about 4 hours while they are passing the meridian. Hence, the LAMOsT is an ideal instrument to perform spectroscopic follow-up for targets of the Kepler mission in an efficient way as it is capable of collecting low-resolution spectra for thousands of objects down to magnitude 17.8 simultaneously. For more detailed information about the LAMOsT, see [9] and [10].

\section{The LAMOST-Kepler project}

In 2010, we initiated the LAMOsT-Kepler project (LK-project) to observe as many objects in the Kepler FoV as possible from the start of the test phase of the LAMost onwards. The main goal is to determine both the stellar parameters and the spectral class of the observed objects in a homogeneous way. Moreover, with low-resolution spectra it is possible to estimate the radial velocity $\left(v_{\text {rad }}\right)$ and the $v \sin i$ (to detect rapid rotation) of the observed objects.

We have composed a prioritised target list consisting of, from high to low priority, $\sim 250$ STANDARD targets (MK secondary standard stars; originally selected for calibration purposes), $\sim 7,000$ KASC targets (scientific interest for the Kepler Asteroseismic Science Consortium), 150,000 PLANET targets (scientific interest for the planet search group), 1,000,000 EXTRA targets (other targets from KIC 10 catalogue; no specific scientific interest), and FIELD targets (objects from the USNO-B catalogue [11]; to fill the fiber holes). As LAMOsT observations can only be optimized for objects with a magnitude interval of $\sim 5$ magnitudes, the targets were subdivided into a bright $(9<K \mathrm{p} \leq 14)$ and faint $(14<K \mathrm{p})$ group. Highest priority was given to objects without KIC 10 parameters. As the temperature determination is the least accurate for hot stars, the others were sorted from high to low KIC $10 T_{\text {eff. }}$ The 
Table 1. For each LK-field, we give the right ascension \& declination of the central bright star and the name of the open cluster that it contains (if applicable). For those that have already been observed up to June 2014, we additionally give the date of observation, the number of plates that were used to observe the LK-field, the total number of LAMOST spectra and the number of different Kepler targets (STANDARD + KASC + PLANET + EXTRA) and field targets that were observed.

\begin{tabular}{llllclrrr}
\hline LK-field & RA(2000) & DE(2000) & Cluster & Date & $\#$ & Spectra & Kepler & Field \\
\hline LK01 & $19: 03: 39.258$ & $+39: 54: 39.24$ & & $30 / 05 / 2011$ & 2 & 1,107 & 1,107 & 0 \\
& & & & $08 / 06 / 2011$ & 2 & 966 & 963 & 3 \\
& & & $02 / 06 / 2014$ & 1 & 3,248 & 3,246 & 2 \\
LK02 & $19: 36: 37.977$ & $+44: 41: 41.77$ & NGC6811 & $04 / 06 / 2012$ & 1 & 563 & 462 & 101 \\
LK03 & $19: 24: 09.919$ & $+39: 12: 42.00$ & NGC6791 & $15 / 06 / 2012$ & 3 & 7,602 & 7,531 & 71 \\
LK04 & $19: 37: 08.862$ & $+40: 12: 49.63$ & NGC6819 & $17 / 06 / 2012$ & 3 & 9,629 & 9,492 & 137 \\
LK05 & $19: 49: 18.139$ & $+41: 34: 56.85$ & & $05 / 10 / 2013$ & 2 & 6,400 & 6,323 & 77 \\
& & & & $22 / 05 / 2014$ & 1 & 1,525 & 1,525 & 0 \\
LK06 & $19: 40: 45.383$ & $+48: 30: 45.10$ & & $22 / 05 / 2013$ & 1 & 394 & 394 & 0 \\
& & & & $23 / 05 / 2013$ & 1 & 1,834 & 1,834 & 0 \\
& & & $14 / 09 / 2013$ & 1 & 2,823 & 2,807 & 16 \\
LK07 & $19: 21: 02.816$ & $+42: 41: 13.07$ & & $19 / 05 / 2013$ & 1 & 1,936 & 1,936 & 0 \\
& & & $26 / 09 / 2013$ & 1 & 3,004 & 2,955 & 49 \\
LK08 & $19: 59: 20.425$ & $+45: 46: 21.15$ & NGC6866 & $02 / 10 / 2013$ & 1 & 2,807 & 2,771 & 36 \\
& & & & $17 / 10 / 2013$ & 1 & 2,592 & 2,565 & 27 \\
& & & & $25 / 09 / 2013$ & 2 & 5,574 & 5,521 & 53 \\
LK09 & $19: 08: 08.340$ & $+44: 02: 10.88$ & & $25 / 10 / 2013$ & 1 & 2,791 & 2,764 & 27 \\
LK10 & $19: 23: 14.829$ & $+47: 11: 44.80$ & & $04 / 10 / 2013$ & 1 & 3,118 & 3,116 & 2 \\
LK11 & $19: 06: 51.499$ & $+48: 55: 31.77$ & & $20 / 05 / 2014$ & 2 & 2,389 & 2,388 & 1 \\
LK12 & $18: 50: 31.041$ & $+42: 54: 43.72$ & & $07 / 10 / 2013$ & 1 & 2,741 & 2,711 & 30 \\
LK13 & $18: 51: 11.993$ & $+46: 44: 17.52$ & & $02 / 05 / 2014$ & 1 & 1,968 & 1,965 & 3 \\
& & & $29 / 05 / 2014$ & 2 & 3,715 & 3,707 & 8 \\
LK14 & $19: 23: 23.787$ & $+50: 16: 16.64$ & & & & & & \\
\hline
\end{tabular}

bright targets were additionally sorted from faint to bright (to avoid saturation + brightest objects can be observed with smaller telescopes) and the faint ones from bright to faint (to avoid underexposure).

We selected 14 LamosT-Kepler fields (LK-fields) to cover the Kepler FoV (Fig. 1, right). Each LKfield contains a central bright star $(V<8)$ for the active optics and four fainter stars $(V<17)$ in the off-centre holes for the guiding of the CCD cameras (cf. Fig. 1, left). A new system for the positioning of the 4,000 fibers of the Lamost has been developed [7] and the code "Survey Strategy System" is used to prepare the observation plans of the LK-project in the most efficient way. More details about the requested LK-fields are given in Table 1. Up to June 2014, all but two of the LK-fields have been observed.

\section{Preliminary results}

There are three teams working on the analysis of the full set of LAmost spectra, each using their own methods.

The Asian team is determining $T_{\text {eff }}, \log g,[F e / H]$ and $v_{\text {rad }}$ with a version of the software package ulyss [12] adapted to the LAmost data (Ren et al., in preparation). It performs a $\chi^{2}$ minimization for a fit of a parametric model to the observed spectra in pixel space. The European team is using an adapted version of the code RotFIT (e.g. [13]) for the same purposes (Frasca et al., in preparation). The observed spectra are fitted to those available in a grid of high-resolution spectra for a selection of more than 1,000 comparison stars with known stellar parameters from the Indo-U.S. Library of Coudé Feed Stellar Spectra [14] after degrading them to the resolution of the LAMosT spectra. As the library 
spectra are in the laboratory rest frame and are corrected for their heliocentric radial velocity, they also serve as templates to derive $v_{\text {rad }}$ with the cross-correlation technique. Moreover, this analysis method is also capable of giving a rough estimation of $v \sin i$ for rapidly rotating stars. The resulting stellar parameters for KASC objects are compared to those derived from available ground-based follow-up spectroscopy obtained with other instruments to check the consistency of the results. $T_{\text {eff }}$ and $v_{\text {rad }}$ can be determined with an accuracy of $\sim 4 \%$ and $\sim 13 \mathrm{~km} \mathrm{~s}^{-1}$, respectively, while $\log g$ can only distinguish between main-sequence and evolved stars. $[\mathrm{Fe} / \mathrm{H}]$ is in general consistent with solar metallicities. Estimates for $v \sin i$ are only relibable for stars rotating faster than $150 \mathrm{~km} \mathrm{~s}^{-1}$.

The American team has developed the code MKcLAss for classifying stars automatically on the MK spectral classification system independent of the stellar parameter determination [15]. This method requires a library of spectral standards and is designed to classify stellar spectra by direct comparison with MK standards using the same criteria as human classifiers. Moreover, MKcLAss is capable of recognizing many of the common spectral peculiarities. For the LAMOsT classifications, the fluxcalibrated standards library with 3.6A-resolution spectra obtained using the GM spectrograph at the Dark Sky Observatory of Appalachian State University is employed. Therefore, the LAmost spectral resolution is slightly degraded to match that of the spectral library. The accuracy of the resulting classifications does not depend upon the accuracy of the flux calibration of the LAmOst spectra. Based on tests on spectra classified by humans, the systematic error and standard deviation of the spectral and luminosity classes are 0.1 and 0.5 spectral subclasses (where a unit spectral subclass is the difference between, for instance, F5 and F6) and 0.02 and 0.5 luminosity classes (where a unit luminosity class is the difference between, for instance, a dwarf (V) and subgiant (IV) classification), respectively. Thus the accuracy of MKCLAss is similar to the level of agreement obtained by two independently working, skilled human classifiers.

\section{Conclusions}

The LK-project is an ambitious observational project that is providing accurate stellar parameters derived from low resolution spectra in an efficient way (4,000 fibers) for objects fainter than most ground-based facilities allow (4-m telescope). The project is not finished yet: two of the requested LK-fields were not observed (scheduled before the end 2014) and not all of LAMOsT spectra have been analysed by the different teams (work in progress). For a full description of the project and its first results, we refer to De Cat et al. (to be submitted to ApJS). The LAmost spectra are available upon request (Peter.DeCat@oma.be).

\section{References}

1. Koch, D. G., Borucki W. J., Basri G., et al., ApJL 713, (2010) L79-L86

2. Cunha M. S., Aerts C., Christensen-Dalsgaard J., et al., A\&A Review 14, (2007) 217-360

3. Latham D. W.,Brown T. M., Monet D. G., et al., AAS 37, (2005) 110.13

4. McNamara B. J., Jackiewicz J., McKeever J., AJ 143, (2012) 101

5. Uytterhoeven K., Briquet M., Bruntt H., et al., AN 331, (2010) 993

6. Wang S.-G., Su D.-Q., Chu Y.-Q., et al., Applied Optics 35, (1996) 5155-5161

7. Xing X., Zhai C., Du H., et al., SPIE 3352, (1998) 839-849

8. Su D. Q., Cui X., Wang Y., Yao Z., SPIE 3352, (1998) 76-90

9. Cui X.-Q., Zhao Y.-H., Chu Y.-Q., et al., RAA 12, (2012) 1197-1242

10. Zhao G., Zhao Y.-H., Chu Y.-Q., et al., RAA 12, (2012) 723-734

11. Monet D. G., Levine S. E., Canzian B., et al., AJ 125, (2003) 984-993

12. Koleva M., Prugniel P., Bouchard A., Wu Y., A\&A 501, (2009) 1269-1279

13. Frasca A., Alcalá J. M., Covino E., et al., A\&A 405, (2003) 149-163

14. Valdes F., Gupta R., Rose J. A., et al., ApJS 152, (2004) 251-259

15. Gray R. O., Corbally C. J., AJ 147, (2014) 80 (7 pages) 